\title{
Experimental and numerical study of non-stationary solid-liquid phase transitions of $n$-tetradecane
}

\author{
Viktoria Zakharova ${ }^{1, *}$, Pavel Kuznetsov ${ }^{1}$, Daniyar Kalimzhanov ${ }^{1}$, and Aleksandr Baranenko ${ }^{1}$ \\ ${ }^{1}$ ITMO University, Faculty of Cryogenic Engineering, 197101, St. Petersburg, Kronverksky pr. 49, Russian Federation
}

\begin{abstract}
During the experiments, four spherical flasks with n-tetradecane with a total mass of $0.344 \mathrm{~kg}$ were placed in a test container inside which an aqueous solution of propylene glycol with a mass concentration of $50 \%$ and an initial temperature of $25{ }^{\circ} \mathrm{C}$ was circulating. As a result of studies, the values of heat flows at different time intervals during the solid-liquid phase transition of n-tetradecane were obtained. Based on the modeling of the processes, the distribution of temperature and velocity of the heat carrier in the test container as well as the values of the local heat transfer coefficients on the surface of the flasks were established. Numerical calculations of the process of phase transition being studied according to the authors' model were also performed. Numerical calculations of the process under study have been performed using the authors' model. A satisfactory convergence of the experimental and calculated values of the heat flow has been obtained. The results can be used in the development of thermal energy accumulators with n-tetradecane.
\end{abstract}

\section{Introduction}

Non-stationary liquid-solid-liquid phase transitions are rather common processes. They can be implemented in various technical devices. In particular, they occur in thermal energy accumulators with phase change materials (PCM) [1, 2].

Thermal energy accumulators are used in heating, hot water supply, cooling and air conditioning systems with variable load. The energy stored in accumulators is used at the maximum consumption of the corresponding resource. This allows installing equipment of lower power, equalizing daily electricity consumption, reducing electricity costs when double-tariff accounting is used and when charging thermal energy storage devices takes place at night. The accumulators can use renewable energy sources such as solar energy and night-time radiation cooling, which reduces energy consumption [3, 4].

In comparison with accumulators with liquid, the advantage of thermal energy accumulators with PCM is that they have an order of magnitude higher volume and mass density of stored energy. This is due to the fact that in them, the main contribution to the thermal energy accumulation is made by the heat of the phase transition.

Also, in such accumulators, the process of accumulating and spending energy stored is carried out at a constant temperature corresponding to the temperature of the phase transition of the PCM. In a more common design of these devices, the PCM is placed inside the container in capsules, which can be of arbitrary shape, including balls or cylinders. The heat carrier circulates in the remaining free internal volume of the accumulator [5].

A fairly large number of theoretical and experimental papers are devoted to the study of processes in such thermal energy storage devices [6-12]. In [6], a detailed experimental and theoretical study of two-dimensional temperature fields in spherical capsules was carried out (axisymmetric problem). In [7], a calculation model for a numerical solution based on Navier - Stokes equation using the enthalpy approach is presented. In [8], the calculation model was applied to study the influence of fins located inside cylindrical capsules on heat exchange. In [9], an experimental and numerical study for elliptical capsules was carried out. However, in these papers, the determination of such quantities as the total heat flow, primarily necessary for the design of cooling systems, occurs indirectly from the results of calculating temperature fields. In [11], the authors investigated the processes in dense backfills of steel capsules. Paper [12] is devoted to modeling a system with thermal energy storage devices as a whole.

For cold accumulation in air conditioning systems, PCM with a phase transition temperature of $5-10{ }^{\circ} \mathrm{C}$ are of interest. Among such substances are paraffins the advantage of which is stability during repeated multidirectional phase transitions, a relatively high specific heat of the phase transition and a slight difference in density in the liquid and solid states.

\section{Research and results}

The authors have performed an experimental and numerical study of the solid-liquid phase transition of n-

* Corresponding author: viktoriasju@yandex.ru 
tetradecane (chemical formula $\mathrm{C}_{14} \mathrm{H}_{30}$ ). According to the passport, the mass fraction of the main substance is $99.89 \%$, the melting point is $6.1{ }^{\circ} \mathrm{C}$. The main properties $[13,14]$ are the following: specific mass heat of melting $\left(\lambda_{\mathrm{hc}}\right)$ is $215 \mathrm{~kJ} / \mathrm{kg}$, liquid phase density $\left(\rho_{\mathrm{hc}}\right)$ at $25{ }^{\circ} \mathrm{C}$ is $762.8 \mathrm{~kg} / \mathrm{m}^{3}$, dynamic viscosity at $25^{\circ} \mathrm{C}$ is $2.13 \cdot 10^{-3} \mathrm{~Pa} \cdot \mathrm{s}$, thermal conductivity coefficient of the liquid phase is $0.143 \mathrm{~W} /(\mathrm{m} \cdot \mathrm{K})$, specific heat of the liquid phase $\left(C_{\mathrm{p} \mathrm{hc}}\right)$ is $2198 \mathrm{~J} /(\mathrm{kg} \cdot \mathrm{K})$.

Experimental studies were carried out in an isolated test container with dimensions of $240 \times 150 \times 115 \mathrm{~mm}$ (Fig. 1). The PCM was placed in round-bottom flasks of a spherical shape with an outer diameter of $66 \mathrm{~mm}$, the flasks were filled to the base of the neck. In the experiments, 4 flasks were used; in them, the PCM mass was $0.344 \mathrm{~kg}$. In the test container, the flasks were located in a strictly defined order in all the experiments as it is shown in Fig. 1. The heat carrier movement was carried out in a closed circuit and with a mass flow rate of $2.1 \mathrm{l} / \mathrm{m}$. The heat carrier volume in the circulation circuit was 4.151 . An aqueous solution of propylene glycol with a mass concentration of $50 \%$ was used as a heat carrier. In all the experiments, the initial temperature of the heat carrier was $25^{\circ} \mathrm{C}$.

The movement of the heat carrier in the test container is shown in Fig. 2, it is modeled in the Fluent package. Modeling is carried out for the steady-state operation mode of the stand. When modeling the movement of the heat carrier, the natural convective movement of the liquid is also considered, since preliminary calculations have shown its noticeable influence in this design of the stand. When modeling, the external walls of the test container are assumed to be thermally insulated, since the temperature of the heat carrier corresponds to the ambient temperature, which eliminates the heat flow between them. The temperature of the walls of the flasks is assumed to be constant and equal to the melting temperature of the SPT.

Before starting the experiments, the flasks with the PCM were cooled until the complete transition of the PCM to a solid state. In the course of these experiments, the following parameters were determined and recorded. At the beginning and end of the experiment, the temperature of the PCM as well as of the phase transition; the heat carrier temperature in regular time intervals, the time of the beginning and end of the experiment, the heat gain from the pump. The experiments were over with the complete transition of the PCM to a liquid state. To measure the temperature, electronic thermometers with an accuracy of $0.2^{\circ} \mathrm{C}$ were used. In the experiments, the phase transition temperature of the PCM was $6.1^{\circ} \mathrm{C}$, which corresponds to its passport value.

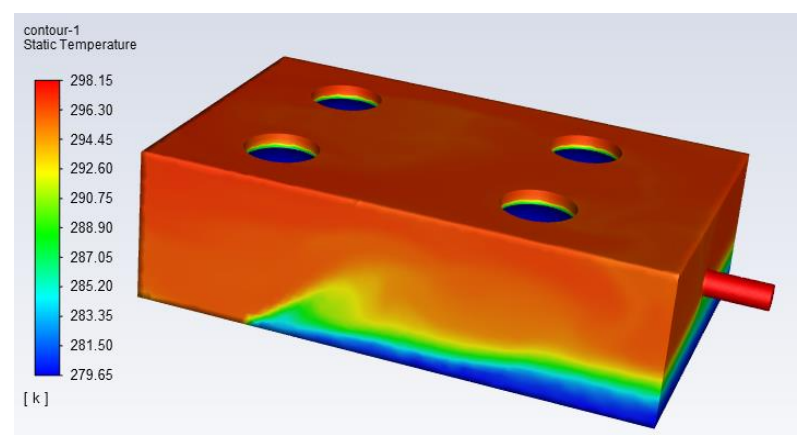

Fig. 1. Temperature distribution in the test container during the experiment.

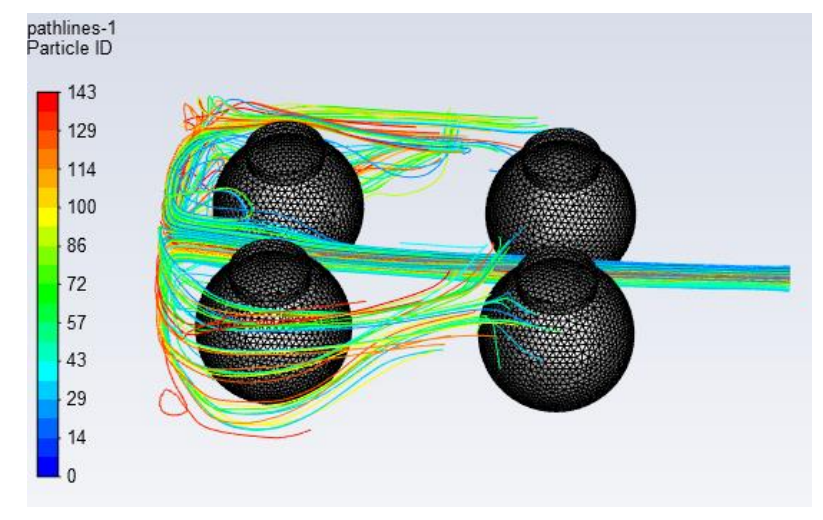

Fig. 2. Heat carrier movement in the test container.

Numerical calculations have also shown that in the test container, during the experiment, the temperature gradient of the heat carrier can reach $10^{\circ} \mathrm{C}$. The lowest temperatures of the heat carrier are set in the lower part of the test container, despite the fact that the heat carrier moves in it at a certain speed. This indicates the feasibility of supplying the heat carrier from below. Local heat transfer coefficients differ by an order of magnitude (Fig. 3). Their maximum value is 3600 $\mathrm{W} /(\mathrm{m} 2 \cdot \mathrm{K})$, the average value is $380 \mathrm{~W} /(\mathrm{m} 2 \cdot \mathrm{K})$. The maximum values of the local heat transfer coefficients take place on the side of the flasks along the flow of the heat carrier of their inlet pipe in the central zone of the test container.

Table 1. Average heat transfer coefficients from flasks to theheat carrier.

\begin{tabular}{|l|c|c|c|c|}
\hline Flask number & 1 & 2 & 3 & 4 \\
\hline $\begin{array}{l}\text { Average heat transfer coefficient, } \\
\mathrm{W} /\left(\mathrm{m}^{2} \cdot \mathrm{K}\right)\end{array}$ & 345 & 453 & 341 & 384 \\
\hline
\end{tabular}

When processing experimental data, the convergence of thermal balances was checked. The heat absorbed by the PCM $\left(Q_{\mathrm{PCM} 1}\right)$ should be equal to the heat released from the heat carrier $\left(Q_{\mathrm{PCM} 2}\right) . Q_{\mathrm{PCM} 2}$ includes the heat of the heat carrier $\left(Q_{\mathrm{hc}}\right)$, flask $\left(Q_{\mathrm{f}}\right)$ and pump $\left(Q_{\mathrm{p}}\right), \mathrm{kJ}$. 


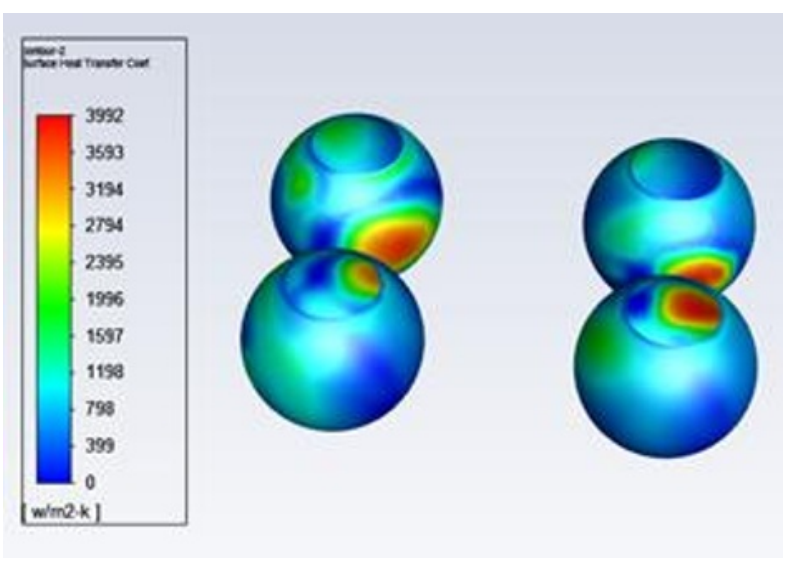

Fig. 3. Local heat transfer coefficients on the surface of the flasks.

$$
Q_{\mathrm{PCM} 1}=Q_{\mathrm{PCM} 2},
$$

where $m_{\mathrm{PCM}}$ is the mass of the SPT in flasks.

In general, the heat of the PCM is determined by the formula

$$
Q_{\mathrm{PCM} 1}=m_{\mathrm{PCM}}\left(C_{\mathrm{p} \mathrm{PCM}} \cdot \Delta t_{\mathrm{PCM}}+\lambda_{\mathrm{PCM}}\right) .
$$

The heat from the heat carrier side

$$
Q_{\mathrm{PCM} 2}=Q_{\mathrm{hc}}+Q_{\mathrm{f}}+Q_{\mathrm{p}} .
$$

The heat of the heat carrier

$$
Q_{\mathrm{hc}}=V_{\mathrm{hc}} \rho_{\mathrm{hc}} C_{\mathrm{phc}} \Delta t_{\mathrm{hc}} \cdot
$$

$V_{\mathrm{hc}}$ is the heat carrier volume in the circulation circuit, $\mathrm{m}^{3}$.

The heat of the flask

$$
Q_{\mathrm{f}}=m_{\mathrm{f}} C_{\mathrm{pf}} \Delta t_{\mathrm{f}} .
$$

where $m_{\mathrm{f}}, C_{\mathrm{pf}}$ are the mass and specific heat of flasks, respectively, $\Delta t_{P C M}, \Delta t_{h c}, \Delta t_{f}$ are temperature differences of the PCM, heat carrier and flask, respectively, at the end and at the beginning of the experiment, ${ }^{\circ} \mathrm{C}$.

The heat of the pump

$$
Q_{\mathrm{p}}=q_{\mathrm{p}} \Delta \tau_{\mathrm{e}}
$$

$q_{\mathrm{p}}$ is the heat flow from the pump, kW. $\Delta \tau_{\mathrm{e}}$ is the experiment conduction time, $\mathrm{s}$.

The heat flow from the pump was determined as follows. The heat carrier circulation was carried out in a closed circuit in the absence of flasks with the SPT in the test container. The initial and final heat carrier temperature and the time of the test were recorded. Knowing the volume of the heat carrier in the circulation circuit, the increase in the temperature of the heat carrier and the duration of the measurement, the amount of heat flow from the pump to the heat carrier was determined. The measurements were made at least three times during each series of experiments. The discrepancy in $q_{\mathrm{p}}$ values did not exceed $5 \%$.

The convergence of thermal balances was $\pm 4 \%$.

The average heat flow from the heat carrier for a certain time interval, $\mathrm{kW}$

$$
\begin{gathered}
q=q_{\mathrm{hc}}+q_{\mathrm{f}}+q_{\mathrm{p}} \\
q_{\mathrm{hc}}=\frac{V_{\mathrm{hc}} \rho_{\mathrm{hc}} C_{\mathrm{phc}} \Delta t_{\mathrm{hc}}}{\Delta \tau_{\mathrm{i}}}, \quad q_{\mathrm{f}}=\frac{m_{\mathrm{f}} C_{\mathrm{pf}} \Delta \mathrm{t}_{\mathrm{f}}}{\Delta \tau_{\mathrm{i}}}
\end{gathered}
$$

$\Delta t_{\mathrm{i}}$ is the change in temperature of the heat carrier and flask for a certain time interval $\Delta \tau_{\mathrm{i}}$.

The values of $q$ calculated from experimental data are presented in Fig. 4. This figure also shows the calculated dependence $q_{\mathrm{PCM}}=f(\tau)$ computed by the authors' model [15].

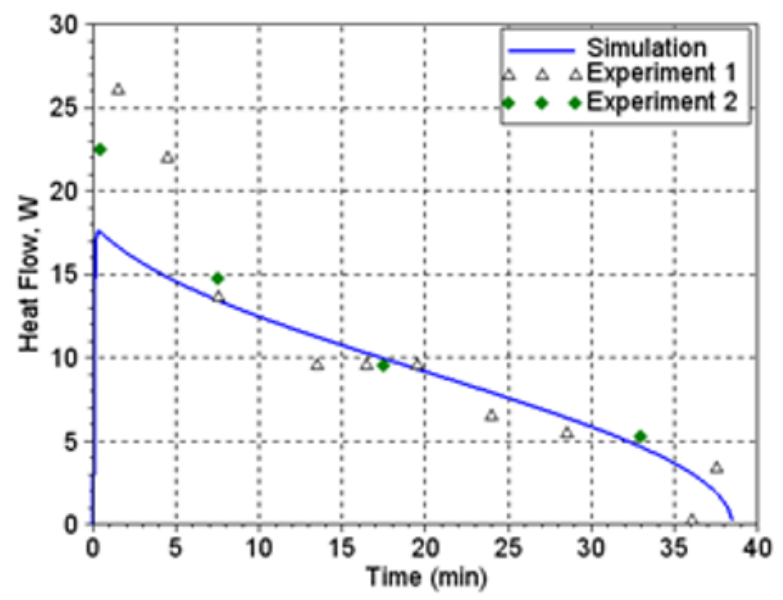

Fig. 4. The average calculated and experimental values of heat flow per flask.

At the initial moment of time, the heat flow increases sharply and after a fairly short time, it begins to decrease due to an increase in the thermal resistance of the liquid phase of the PCM (since its thickness increases) and a decrease in the contact surface of the phases of the PCM. The results obtained correspond to the regularities of the process under study as well as to the data of other authors [16]. At the same time, the calculated and experimental values of the duration of the solid-liquid phase transition process differ by only $2 \%$. The greatest interest present the data on the values of the heat flow in the time interval of the phase transition process of 5-30 min, when the dependence of $q_{\mathrm{p}}$ on time is monotonous. Based on these data, it is most advisable to model the calculations of thermal energy accumulators.

\section{Conclusions}

The duration of the experiment was 36-38 minutes. The heat flow reached its maximum value of $26 \mathrm{~W}$ in 90 seconds after the start of the experiments. In the interval of 5-30 minutes, the dependence of the heat flow on time is monotonous. The results obtained by the authors have confirmed the adequacy of the previously developed 
model of the phase transition process. They can be used in the development of methods for calculating thermal energy accumulators with the PCM.

\section{References}

[1] C. Veerakumar, A. Sreekumar, Int J Refrig 67, (2016)

[2] B. Al-Aifan, R. Parameshwaran, M. Kushagra, R. Karunakaran, Int J Refrig 76 (2017)

[3] G.T. Kim, Y.U. Choi, Y. Chung, M.S. Kim, K.W. Park, M.S. Kim, Int J Refrig 88 (2018)

[4] L. Mishra, A. Sinha, R. Gupta, Green Buildings and Sustainable Engineering, Proceedings of GBSE 2018 (2019)

[5] A. Sharma, V.V. Tyagi, C.R. Chen, D. Buddhi, Renew. Sust. Energ. Rev. 13 (2009)

[6] F.L. Tan, S.F. Hosseinizadeh, J.M. Khodadadi, L. Fan, Int. J. Heat Mass Transf. 52 (2009)

[7] H. Sattari, A. Mohebbi, M.M. Afsahi, A.A. Yancheshme, Int J Refrig 73 (2017)

[8] M.M. Joybari, F. Haghighat, S. Seddegh, A.A. Al-Abidi, Energy Convers. Manag. 152 (2017)

[9] D.H. Shina, J. Parka, S.H. Choib, H.S. Kob, S.W. Karnga, Y. Shina, Energy Convers. Manag. 182 (2019)

[10] M.A.M. Rosli, Renew. Sust. Energ. Rev. 42, (2015)

[11] G. Zsembinszki, G.F. Angel, L.F. Cabeza, Appl. Sci 10, (2020)

[12] R. Gulfam, W. Zhu, L. Xu, I.I. Cheema, P. Sheng, G. Zhao, Y.D. Deng, Energy Convers. Manag. 156, (2018)

[13] T.S. Khasanshin, A.P. Shchemelev, High Temp. 40, (2002)

[14] D. Mackay, et al., Handbook of physical-chemical properties and environmental fate for organic chemicals, 2nd ed (New York, 2006)

[15] V.Y. Zakharova, P.A. Kuznetsov, A.V. Baranenko, IOP Conference Series: Mater. Sci. Eng. 643, 1 (2019)

[16] N. Khandelwal1, A. Gupta, A. Tripathi, A. Pal, A. Jha, Our Heritage Journal 22, 1 (2020) 